\begin{tabular}{ccc}
\hline & International Journal of Engineering \& Technology, $7(2.28)(2018) 362-368$ \\
SPC & International Journal of Engineering \& Technology \\
Website: www.sciencepubco.com/index.php/IJET & Research paper \\
\hline
\end{tabular}

\title{
Power factor control of PV generators in local distribution networks using fuzzy logic concept
}

\author{
Raed A. Shalwala * \\ Umm Alqura university \\ *Corresponding author E-mail: rashalwala@uqu.edu.sa
}

\begin{abstract}
One of the most important operational requirements for any electrical power network for both distribution and transmission level is voltage control. Many studies have been carried out to improve or develop new voltage control techniques to facilitate safe connection of distributed generation. In Saudi Arabia, due to environmental, economic and development perspectives, a wide integration of photovoltaic (PV) genera-tion in distribution network is expected in the near future. This development in the network may cause voltage regulation problems due to the interaction with the existing conventional control system. In a previous paper, a control system has been described using a fuzzy logic control to set the on-line tap changer for the primary substation. In this paper a new control system is proposed for controlling the power factor of individual PV invertors based on observed correlation between net active and reactive power at each connection. A fuzzy logic control has been designed to alter the power factor for the remote invertors from the secondary substation to keep the feeder voltage within the permissible limits. In order to confirm the validity of the proposed method, simulations are carried out for a realistic distribution network with real data for load and solar radiation. Results showing the performance of the new control method are presented and discussed.
\end{abstract}

Keywords: Grid Connected PV Syste;, Distribution Network, Power Factor; Fuzzy Logic; Control

\section{Introduction}

This paper presents an approach for solving the feeder voltage regulation problem in a local manner, with the goal of fulfilling the plug-and-play feature desired by manufacturers and regulatory bodies. The plug-and-play feature will enable customers to simply connect their PV systems to the distribution feeder, and through a fuzzy logic control (FLC) the power flow from the PV system through the inverters will be controlled to help maintain the feeder voltage level within limits at minimum cost. The proposed controller design is based on FLC and the architecture is then presented. Test results of this system are shown to prove that the proposed controller can successfully regulate the voltage of a distribution feeder with PV systems connected to it. Finally the interaction between two controllers at the same feeder has been investigated and a simulation is carried out under MATLAB /Simulink environment to evaluate the stability of the control algorithm.

\section{Control of the reactive power of PV genera- tion using FLC}

Consider a voltage source $\mathrm{V}_{\mathrm{S}}\left\llcorner\delta\right.$ connected to a utility grid $\mathrm{V}_{\mathrm{G}}\left\llcorner 0^{\circ}\right.$ through a coupling impedance $\mathrm{Z}=\mathrm{R}+\mathrm{j} \mathrm{X}$, as shown in Fig.1.

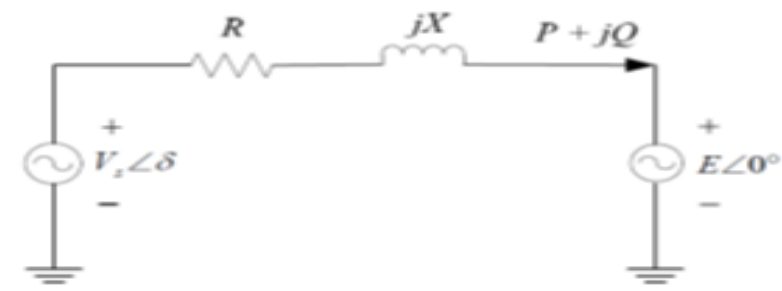

Fig. 1: Power Flow between A Voltage Source and Utility Grid.

The real and reactive power delivered to the utility grid is [2]:

$\mathrm{P}=\frac{\mathrm{V}_{\mathrm{G}} \mathrm{V}_{\mathrm{S}}}{\mathrm{Z}} \cos \left(\theta_{\mathrm{Z}}-\delta\right)-\frac{\mathrm{V}_{\mathrm{G}}^{2}}{\mathrm{Z}} \cos \left(\theta_{\mathrm{Z}}\right)$
$\mathrm{Q}=\frac{\mathrm{V}_{\mathrm{G}} \mathrm{V}_{\mathrm{S}}}{\mathrm{Z}} \sin \left(\theta_{\mathrm{Z}}-\delta\right)-\frac{\mathrm{V}_{\mathrm{G}}^{2}}{\mathrm{Z}} \sin \left(\theta_{\mathrm{Z}}\right)$

From eq. 1 and eq.2, it is clear that the real and reactive power delivered to the utility grid are completely determined by the amplitude and angle of the sending voltage source, i.e. the output voltage of the inverter. On the other hand, the values of $V_{S}$ and $\delta$ can be determined from eq. 3 and eq. 4 if the desired values of real and reactive power are given.

$$
\begin{aligned}
& \mathrm{V}_{\mathrm{S}}=\left[\frac{\mathrm{Z}^{2}}{\mathrm{~V}_{\mathrm{G}}^{2}}\left(\mathrm{P}^{2}+\mathrm{Q}^{2}\right)+\mathrm{V}_{\mathrm{G}}^{2}+2 \mathrm{PZ} \cos \left(\theta_{\mathrm{Z}}\right)+2 \mathrm{QZ} \sin \left(\theta_{\mathrm{Z}}\right)\right]^{1 / 2} \\
& \delta=\theta_{\mathrm{Z}}-\cos ^{-1}\left[\frac{\mathrm{ZP}}{\mathrm{V}_{\mathrm{G}} \mathrm{V}_{\mathrm{S}}}+\frac{\mathrm{V}_{\mathrm{G}}}{\mathrm{V}_{\mathrm{S}}} \cos \left(\theta_{\mathrm{Z}}\right)\right]
\end{aligned}
$$

When the PV generator is connected to the radial feeder, its active power export reduces the power flow from the secondary substation. 
This causes reduction in the voltage drop along the feeder. If the generator's power export is larger than the feeder load, power flows from the generator to the primary substation and this causes a voltage rise along the feeder.

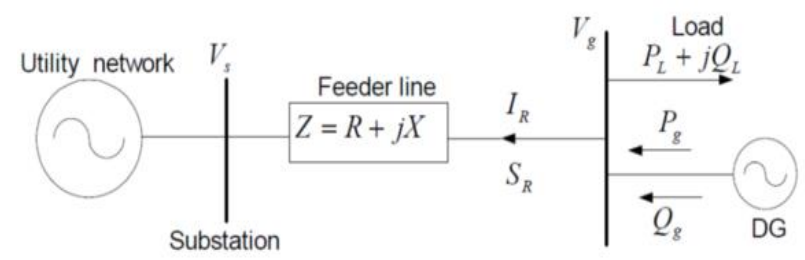

Fig. 2: Utility Network with DG.

Eq.1 describes how the voltage level is changed at the point of connection of the DG. The active power produced by DG increase the voltage, whereas the reactive power can further increase or reduce it depending on the type of DG technology. The PV generator can generate or absorb reactive power depending on the operating power factor of the inverter. These outcomes, in combination with the system's R/ X ratio or distribution network characteristics and load profiles, determine the increase in voltage level at the connection point due to power production of the PV generator.

The inverter can only dispatch or consume the reactive power if its apparent power capability (S) exceed the instantaneous power generated by the connected PV panels. The range of allowable reactive power consumption or generation is given by eq.5.

$|\mathrm{Q}| \leq \sqrt{ }\left(\mathrm{S}^{\wedge} 2-\llbracket \mathrm{P} \_\mathrm{PV} \rrbracket \wedge 2\right)$

In this study the inverter is assumed to be operating up to 0.9 lagging power factor.

\section{Design of FL power factor controller for in- verter}

According to eq. 3 the active power generated by the PV and the active power consumed by the load will be used as an input to the fuzzy logic controller. Also, the distance from the secondary substation will be used as third input. The operating power factor for the inverter will be the output of the controller. The customer feeder I9 in the typical model of the distribution network in Fig.7 will be considered for investigating the behavior of the controller under different conditions of load and solar irradiation.Fig.3 shows the step by step design for the FL power factor controller for inverter.

\subsection{Input membership functions}

\subsubsection{Output power of $P V\left(P_{P V}\right)$}

The output power of PV generator membership is represented by a range of $0 \mathrm{~kW}$ to $40 \mathrm{~kW}$.

Table 1: Membership Function P_PV Range

\begin{tabular}{lll}
\hline MF & & Approximate range $(\mathrm{kW})$ \\
\hline L & Low & $0-9$ \\
M & Medium & $9-24$ \\
H & High & $24-40$ \\
\hline
\end{tabular}

The triangular membership function is used to take advantage of the simplicity of the system and reduce the running time of the program for the whole integrated fuzzy system.

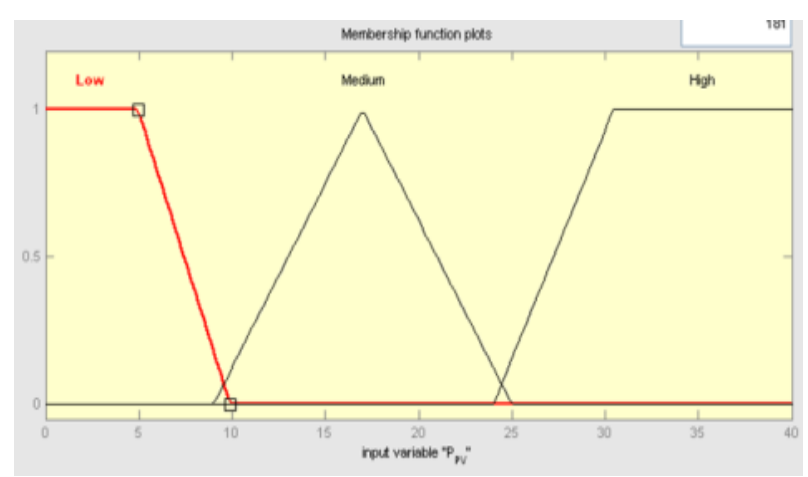

Fig. 3: Input Membership Function $\mathbf{P}_{\mathbf{P V}}$

\subsubsection{Load power $P_{L}$}

The load power of each node membership is represented by a range of $0 \mathrm{~kW}$ to $20 \mathrm{~kW}$.

\begin{tabular}{lll} 
& \multicolumn{2}{c}{ Table 2: Membership Function P_L Range } \\
\hline MF & & Approximate range $(\mathrm{kW})$ \\
\hline L & Low & $0-7.5$ \\
M & Medium & $7.5-16.5$ \\
H & High & $16.2-22$ \\
\hline
\end{tabular}

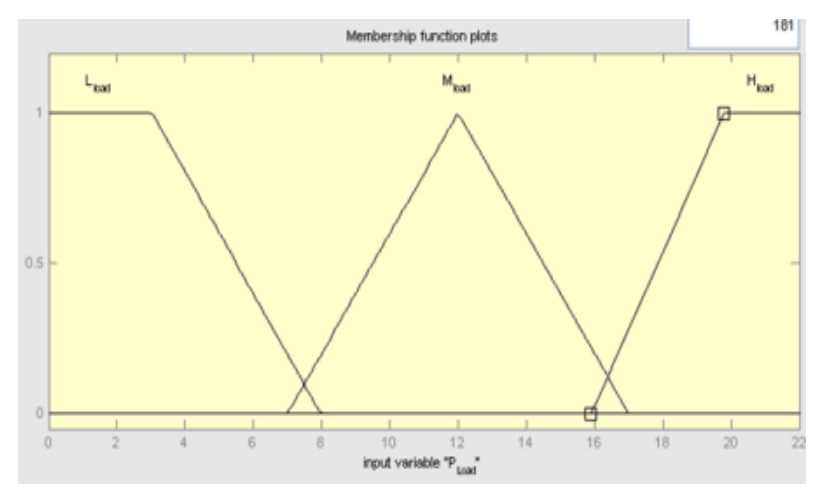

Fig. 4: Input Membership Function P_L.

\subsubsection{Distance from secondary substation}

The distance for each node from substation membership is presented by a range $0 \mathrm{~m}$ to $200 \mathrm{~m}$ according to the base model.

Table 1: Membership Function D Range

\begin{tabular}{lll}
\multicolumn{2}{c}{ Table 1: Membership Function D Range } \\
\hline MF & & Approximate range $(\mathrm{m})$ \\
\hline $\mathrm{N}$ & Near & $0-50$ \\
$\mathrm{M}$ & Medium & $50-100$ \\
$\mathrm{~F}$ & Far & $100-200$ \\
\hline
\end{tabular}

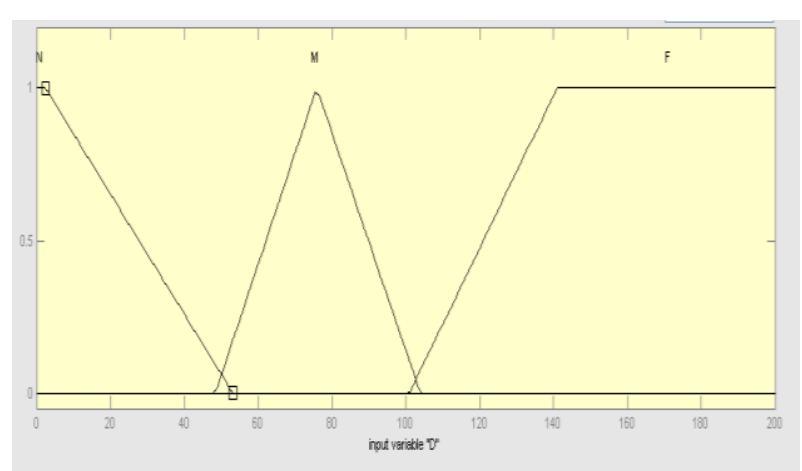

Fig. 5: Input Membership Function D.

\subsection{Output membership function}




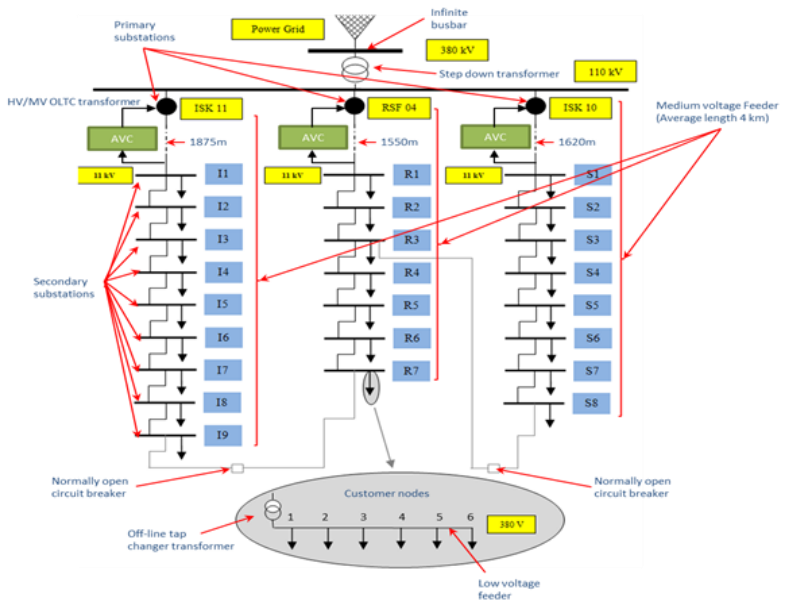

Fig. 6: Typical Distribution Network.

Overcoming the voltage rise issue at any node of the feeder with PVs can be done by operating the PV generators at 0.9 lagging power factor. This value will be used for the far nodes in the worst case scenario with maximum PV and no load. Fig.8 shows the voltage profile along the $\mathrm{I} 9$ feeder with maximum $\mathrm{PV}$ at node 5 and 6 and no load at all nodes.

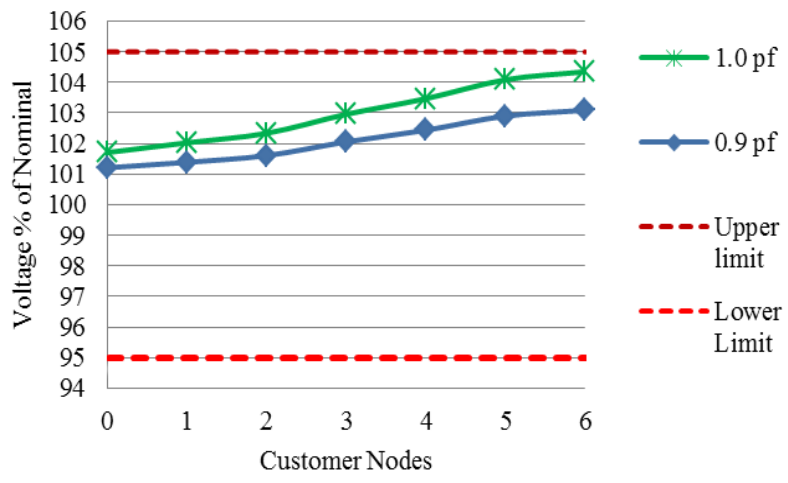

Fig. 7: Voltage Profile of I9 (No Load \& Max PV at Node $5 \& 6$ ).

For the medium distance node from the secondary substation 0.95 lagging power factor will be used. Fig.9 shows the voltage profile along the I9 feeder with maximum PV at node 3 and 4 and no load at all nodes.

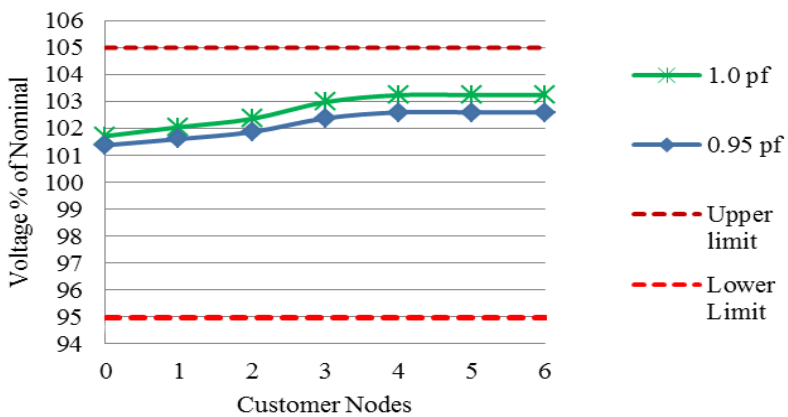

Fig. 8: Voltage Profile I9 (No Load \& Max PV at Node $3 \& 4$ ).

Finally, for the near nodes from the substation the inverter will be kept uncontrolled which means that the inverter operates at unity power factor for all cases. This makes this proposed method cheaper. Fig. 10 shows the voltage profile along the I9 feeder with maximum PV at node [1] and 2 and no load at all nodes.

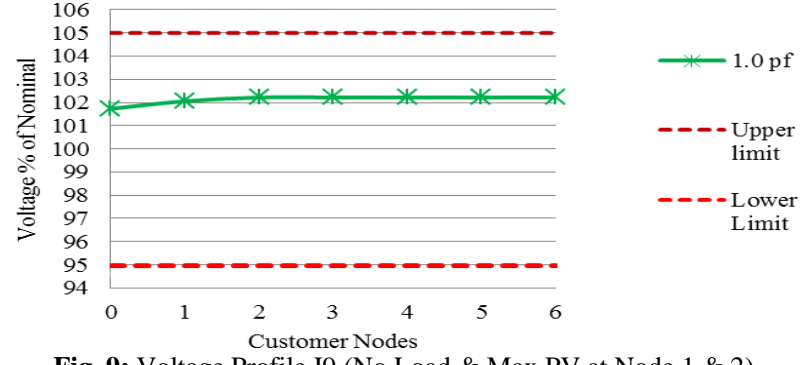

Fig. 9: Voltage Profile I9 (No Load \& Max PV at Node $1 \&$ 2).

In order to force the output of the controller to choose one of these lagging power factors $(0.9$ and 0.95$)$ or keep it working at unity power factor a membership function with a single spike is used. Fig.11 shows the output membership function for the power factor set.

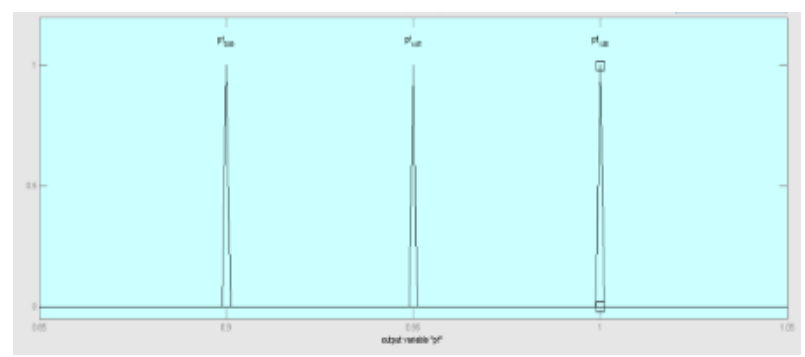

Fig. 10: Output Membership Function Lagging Power Factor Set

\subsection{The control rules}

The possible combination between the 3 inputs situations will give a total number of 27 rules as there are 3 situations for each input. However, because the near nodes will be uncontrolled the total number of rules reduces to 18 rules. The desired power factor will be selected logically for each situation. Table. 4 shows the fuzzy rules to set up the power factor of the inverter at customer in the middle range distance from substation $(50-100 \mathrm{~m})$.

Table 4: Fuzzy Rule Base for Nodes in Medium Range Distance

\begin{tabular}{llll}
\hline PV & Low & Medium & High \\
Load & 1.0 & 1.0 & 0.95 \\
Low & 1.0 & 1.0 & 1.0 \\
Medium & 1.0 & 1.0 & 1.0 \\
High & & & \\
\hline
\end{tabular}

Table.5 shows the fuzzy rules to set up the power factor of the inverter at customer in the far distance from substation (100-200m).

Table 2: Fuzzy Rule Base for Nodes in Far Range Distance

\begin{tabular}{llll}
\hline PV Load & Low & Medium & High \\
\hline Low & 1.0 & 0.95 & 0.90 \\
Medium & 1.0 & 1.0 & 0.95 \\
High & 1.0 & 1.0 & 1.0 \\
\hline
\end{tabular}

Fig.11 shows the proposed FLC for each generator.

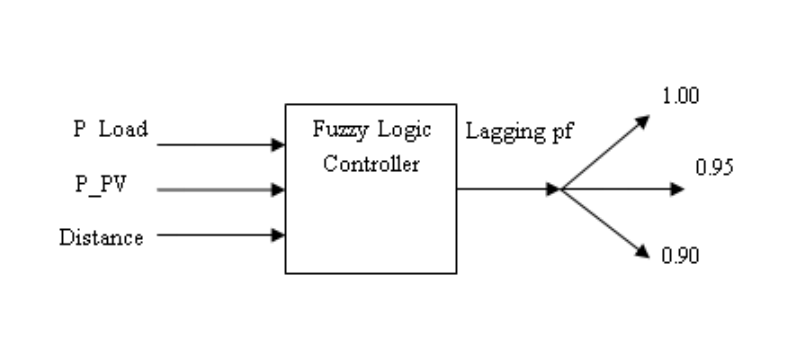

Fig. 11: Proposed FLC. 


\section{Simulations and results}

The steady-state solution has been investigated to check the behavior of the proposed controller at worst case scenarios. For each case the selected data for the load power, the power generated by PV and the distance from the secondary substation are used as inputs for the fuzzy system in MATLAB Simulink environment. The output of this system is used to set the operating pf for generators at each node in 19 feeder by using the ETAP software package. Then, the simulation is run to get the voltage level at each node with the fuzzy logic decision and rerun when the generators are kept uncontrolled. After that, the readings of voltages are taken to an excel spread sheet to draw the voltage profile of both cases:

1) Uncontrolled PV inverter (ie. $1.0 \mathrm{pf}$ ).

2) Controlled PV inverter by FLC.

Table. 6 shows all possible worst case scenarios that can be happen at the feeder.

Table 6: Worst Case Scenarios

\begin{tabular}{|c|c|c|c|c|c|c|}
\hline & \multicolumn{3}{|c|}{$P_{\text {Load }}$} & \multicolumn{3}{|c|}{$P_{P V}$} \\
\hline Node & 1,2 & 3,4 & 5,6 & 1,2 & 3,4 & 5,6 \\
\hline Case & & & & $\operatorname{Max}$ & Max & Max \\
\hline 1 & No & No & No & high & high & high \\
\hline Case & & $\mathrm{No}$ & Min me- & $\operatorname{Max}$ & Max & Max \\
\hline 2 & No & No & dium & high & high & high \\
\hline Case & & Min me- & Min me- & Max & $\operatorname{Max}$ & Max \\
\hline 3 & No & dium & dium & high & high & high \\
\hline Case & $\mathrm{No}$ & Min me- & Min & Max & Max & Max \\
\hline 4 & No & dium & high & high & high & high \\
\hline Case & $\mathrm{No}$ & $\mathrm{No}$ & $\mathrm{No}$ & $\operatorname{Max}$ & $\operatorname{Max}$ & Max \\
\hline 5 & & No & No & high & high & medium \\
\hline $\begin{array}{l}\text { Case } \\
6\end{array}$ & No & No & No & $\begin{array}{l}\text { Max } \\
\text { high }\end{array}$ & $\begin{array}{l}\text { Max } \\
\text { medium }\end{array}$ & $\begin{array}{l}\text { Max } \\
\text { medium }\end{array}$ \\
\hline $\begin{array}{l}\text { Case } \\
7\end{array}$ & No & No & No & $\begin{array}{l}\text { Max } \\
\text { high }\end{array}$ & $\begin{array}{l}\text { Max } \\
\text { high }\end{array}$ & Max low \\
\hline $\begin{array}{l}\text { Case } \\
8\end{array}$ & No & No & No & $\begin{array}{l}\text { Max } \\
\text { high }\end{array}$ & $\begin{array}{l}\text { Max } \\
\text { medium }\end{array}$ & Max low \\
\hline
\end{tabular}

Fig.13 to Fig.20 show the results for all worst case scenarios.

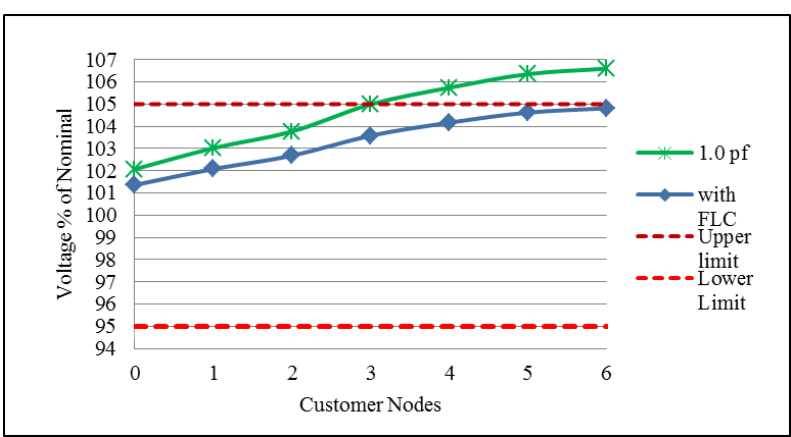

Fig. 12: Case 1: Voltage Profile I9 (No Load \& Max High PV at All Node).

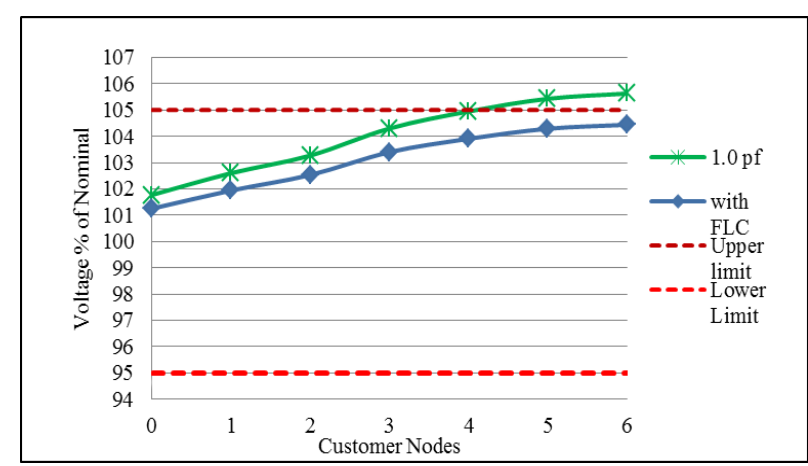

Fig. 13: Case 2: Voltage Profile I9 (Min Medium Load \& Max High PV at Node 5, 6 and No Load \& Max High PV at Node 1, 2, 3, 4).

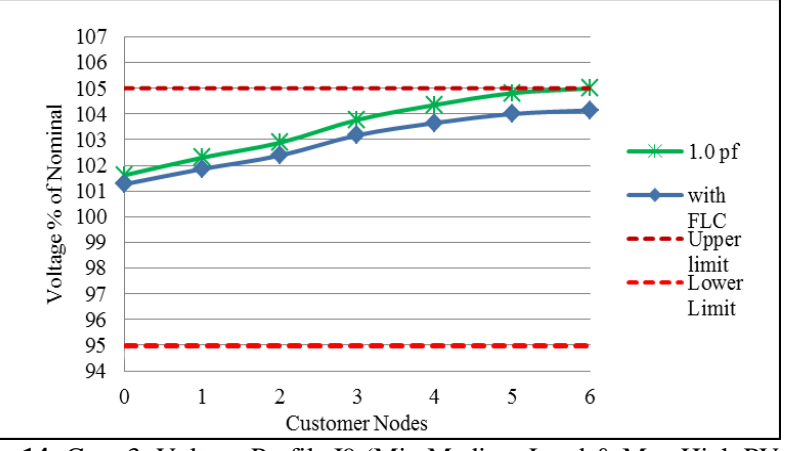

Fig. 14: Case 3: Voltage Profile I9 (Min Medium Load \& Max High PV at Node 3, 4, 5, 6 and No Load \& Max High PV At Node 1, 2)

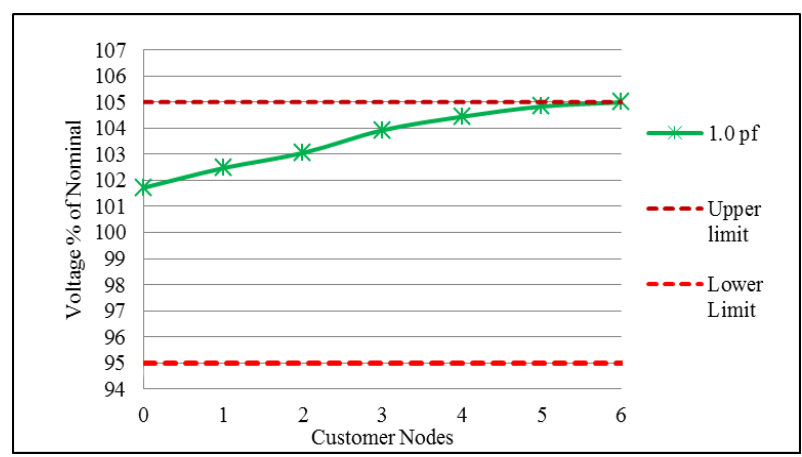

Fig. 15: Case 4: Voltage Profile I9 (Min High Load \& Max High PV at Node 5, 6 and Min Medium Load \& Max High PV At Node 3, 4 and No Load \& Max High PV at Node 1, 2)

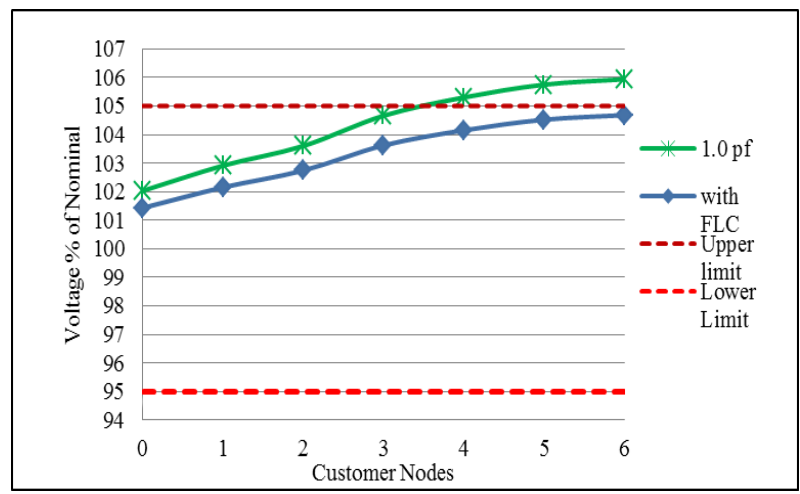

Fig. 16: Case 5: Voltage Profile I9 (No Load \& Max Medium PV at Node 5, 6 and No Load \& Max High PV at Node 1, 2, 3, 4).

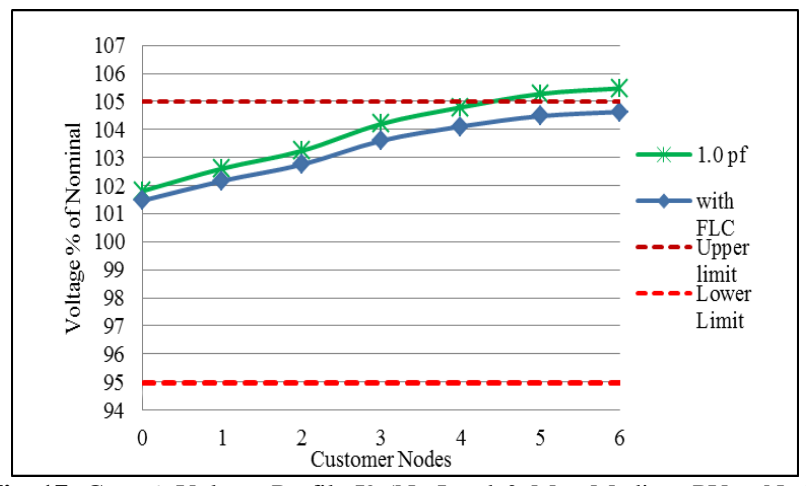

Fig. 17: Case 6: Voltage Profile I9 (No Load \& Max Medium PV at Node 3, 4, 5, 6 and No Load \& Max High PV at Node 1,2). 


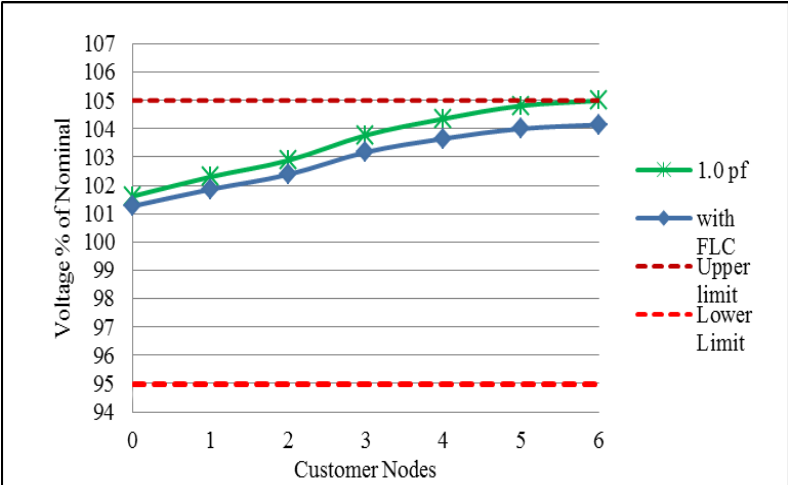

Fig. 18: Case 7: Voltage Profile I9 (No Load \& Max Low PV at Node 5, 6 and No Load \& Max High PV at Node 1, 2, 3, 4)

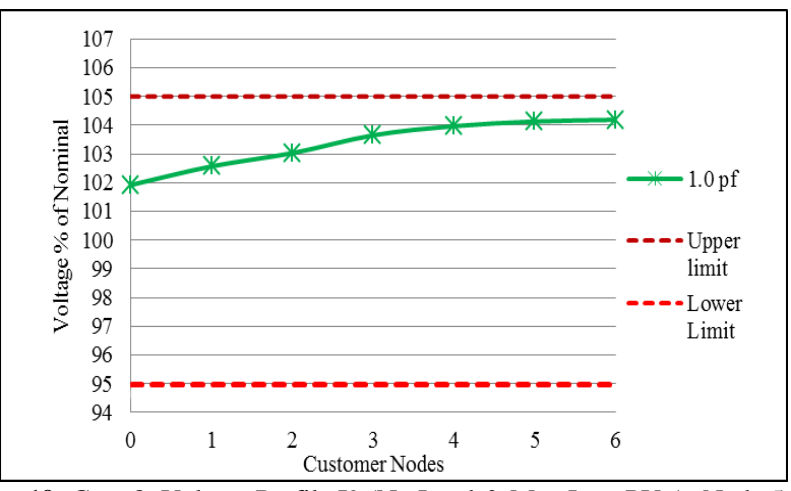

Fig. 19: Case 8: Voltage Profile I9 (No Load \& Max Low PV At Node 5, 6 and No Load and Max Medium PV at Node 3, 4 and No Load \& Max High $\mathrm{PV}$ at Node 1, 2).

The previous figures show clearly the ability of the proposed FLC to keep the voltage level for all nodes within the permissible level. Also, the voltage profile can be brought down for case 4 by resetting the range of the input memberships for medium load if this desired by the network operator. As the proposed FLC would manage to keep the voltage between the limits in all worst cases, it will be valid for all other possible scenarios, meaning that it offers a plug and play option for each customer.

\section{Stability assessment for the proposed FLC}

\subsection{Mat lab/Simulink model}

In order to check the stability of the previous proposed controller for each generator on a same feeder and check the interaction between them, a dynamic simulation is needed with at least two controller at the same feeder. In this section the MATLAB/Simulink with powersys tool box will be used to investigate this issue. The same model designed in [3] to control the inverter for fuel cells by setting the required active and reactive power will be used. Fig. 21 shows the block diagram for the controller of the inverter.

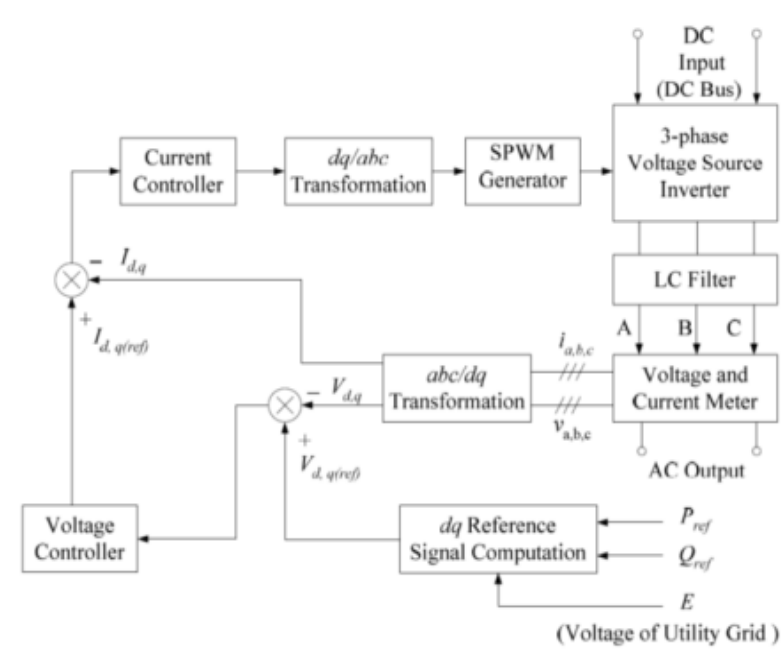

Fig. 20: Block Diagram of the Controller for the Inverter [3].

In Fig.21, the "dq Reference Signal Computation" block, which is based on eq. 3 and eq.4, calculates the magnitude and angle of the filtered output voltages of the inverter and then converts them into $\mathrm{dq}$ voltage reference signals. The "abc/dq Transformation" block takes the current and voltage values (in abc coordinate) from the voltage and current meters and converts them into dq values. The outer voltage controller takes the error signals between the actual output voltage in $\mathrm{dq}$ frame $\left(\mathrm{V}_{\mathrm{d}, \mathrm{q}}\right)$ and the reference voltage $\left(\mathrm{V}_{\mathrm{d}, \mathrm{q}(\mathrm{ref})}\right)$ and generates the current reference signals $\left(\mathrm{I}_{\mathrm{d}, \mathrm{q}(\mathrm{ref})}\right)$ for the current control loop. The inner current controller produces the dq control signals, which are converted back into the control signals in abc coordinates through the "dq/abc Transformation" block. These control signals are used to modulate the sinusoidal pulse width modulation (SPWM) pulse generator to produce the proper pulses for the inverter switches, which control the inverter output voltage.

However, the desired active and reactive power is derived by the proposed FLC. The stability issue for such a system appears because the value of the voltage at the node of connection is taken into account for setting the reactive output power of the inverter. At the same time the voltage level at the node of connection is changed by the total power penetration from the inverter as has been shown before. So, the main objective of this section is to investigate the behaviour of two controllers at two nodes in the same feeder while changing the load and the power from the PV generator. Nodes 5 and 6 of I9 feeder have been taken into consideration with maximum power integration. The load at node 5 will be changed from low to high while the load at node 6 goes from high to low at the same time. All other nodes in the feeder are kept constant and without PV integration. Fig. 22 shows the simulation model for each inverter in MATLAB/Simulink. The output of the fuzzy logic controller (power factor) and the total power generated by PV will be used to calculate the required reactive power by using an embedded function, Qcal in MATLAB/Simulink. Another embedded function, VsD used to calculate V_S and $\delta$ based on eq.3 and eq.4. Then reference values for the voltage control loop (V_(d,q(ref)) ) are calculated by Park's transformation. In addition, the voltage regulator includes the current control loop to set up the pulses for the inverter to deliver the required active and reactive power to the system.

Fig. 23 shows the simulation model for the feeder I9 with the FLC for node 5 and 6. 


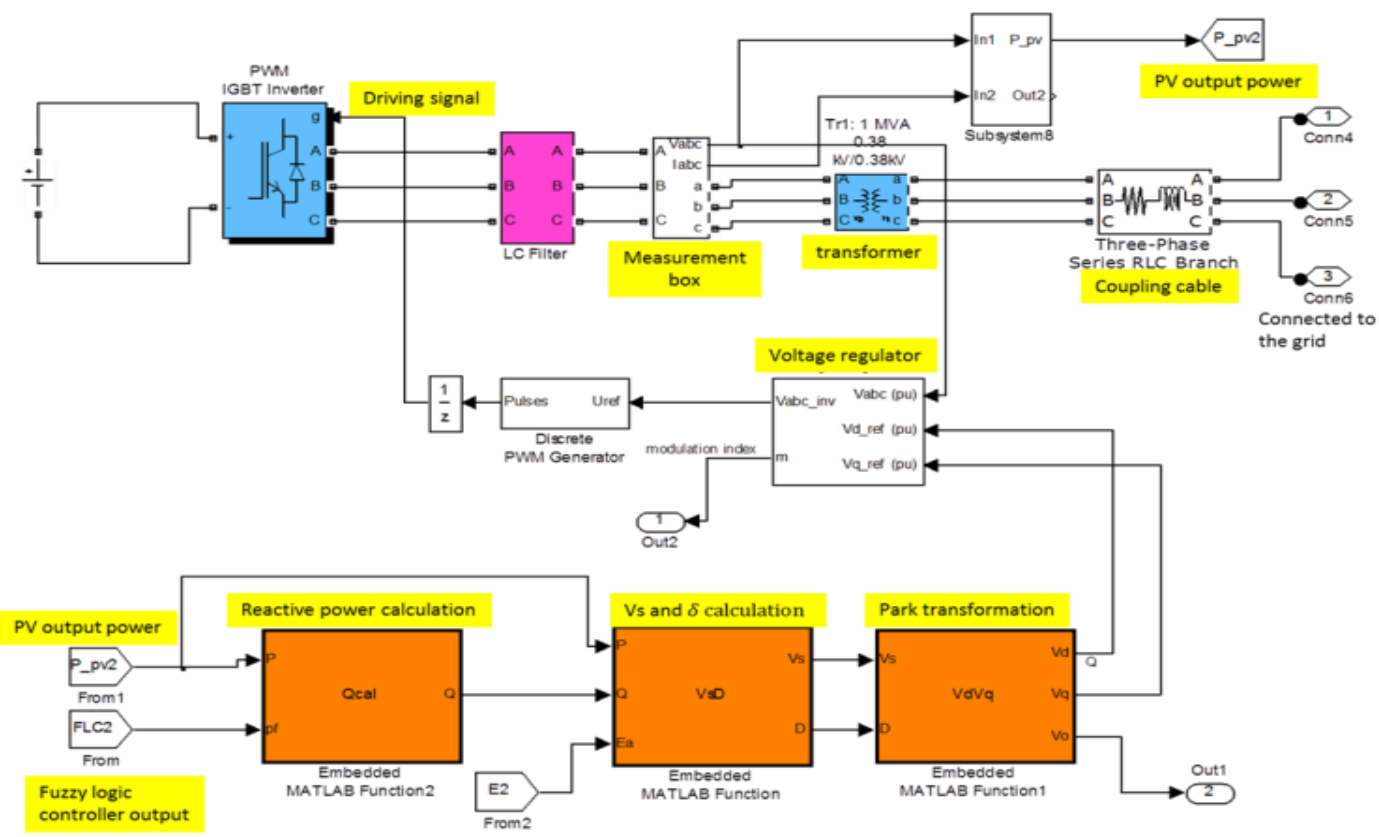

Fig. 21: Controlled Inverter.

The output of the fuzzy logic controller (power factor) and the total power generated by PV will be used to calculate the required reactive power by using an embedded function, Qcal in

MATLAB/Simulink. Another embedded function, VsD used to calculate $V_{\mathrm{S}}$ and $\delta$ based on eq. 3 and eq.4. Then reference values for the voltage control loop ( $\left.\mathrm{V}_{\mathrm{d}, \mathrm{q}(\mathrm{ref})}\right)$ are calculated by Park's transformation. Also, the voltage regulator includes the current control loop to set up the pulses for the inverter to deliver the required active and reactive power to the system.

Fig. 23 shows the simulation model for the feeder I9 with the FLC for node 5 and 6 .

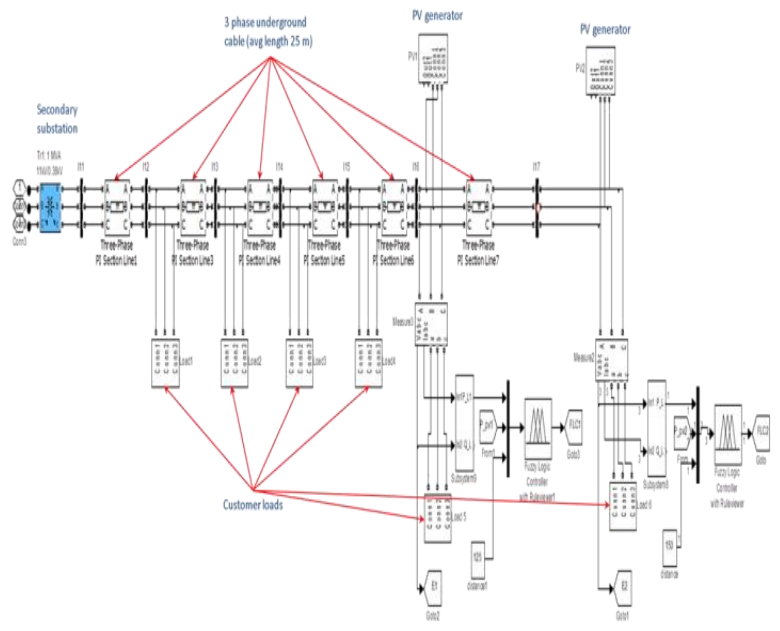

Fig. 22: Simulation Model for I9 Feeder.

\subsection{Results of simulations}

Fig.24 and Fig.25 shows the load and the FLC decision at node 5 and 6 . The PV generators remain at maximum value during the simulation for both nodes.
(A)

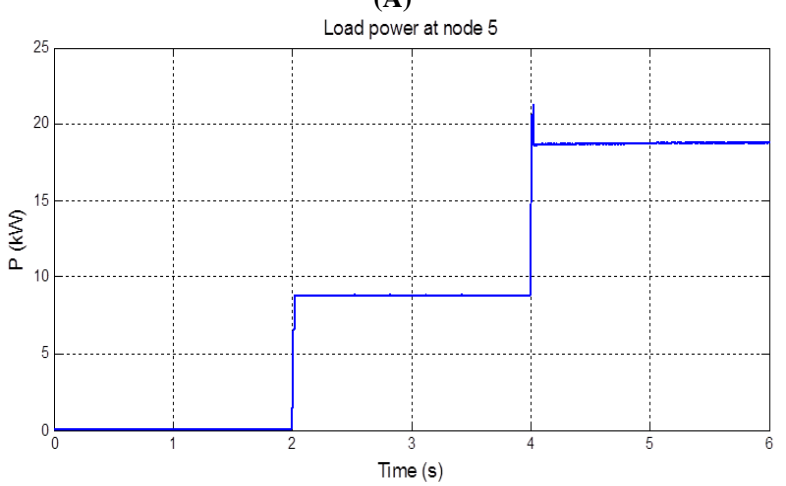

(B)

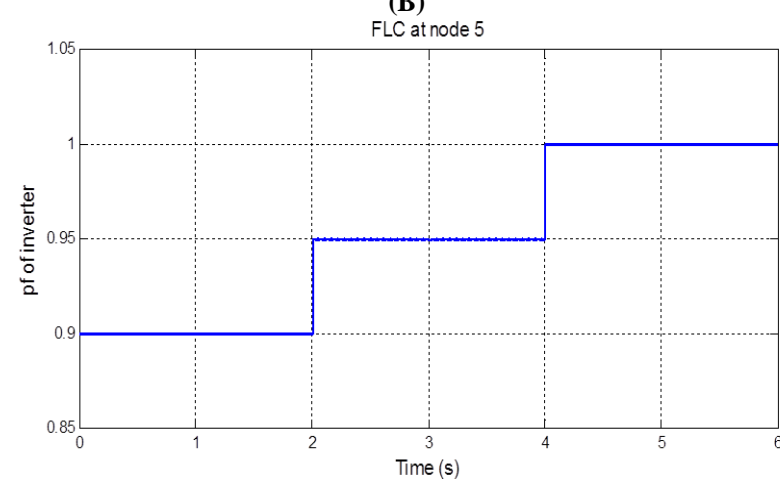

(C)

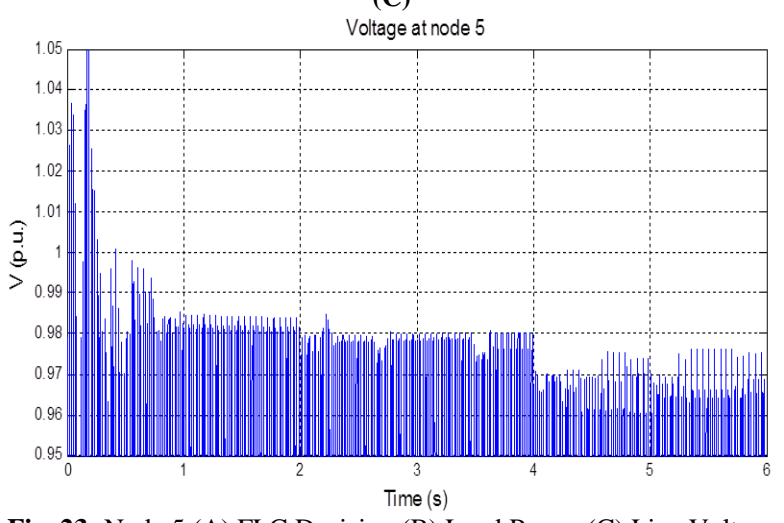

Fig. 23: Node 5 (A) FLC Decision (B) Load Power (C) Line Voltage 
(A)

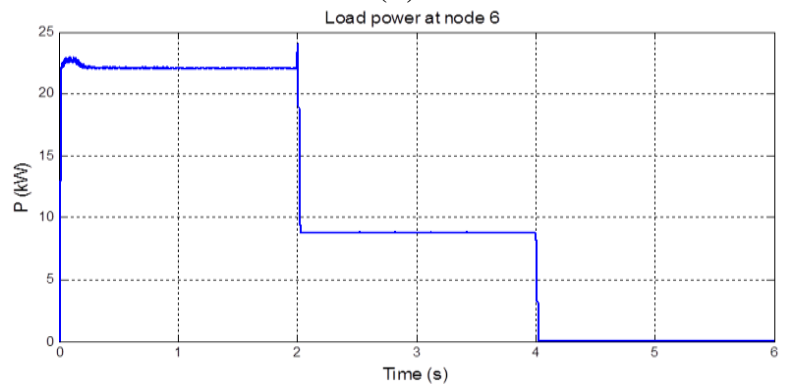

(B)

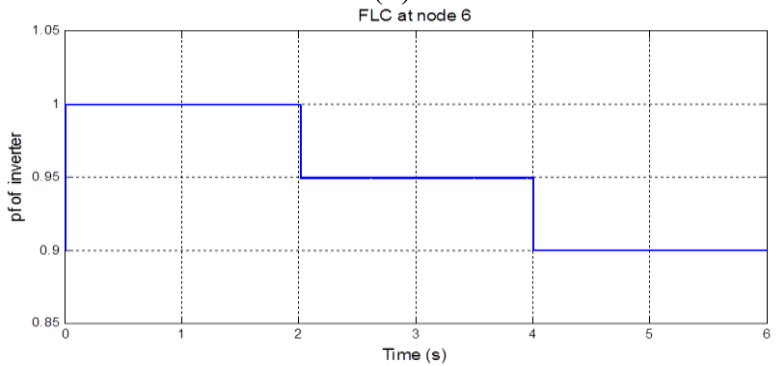

(C)

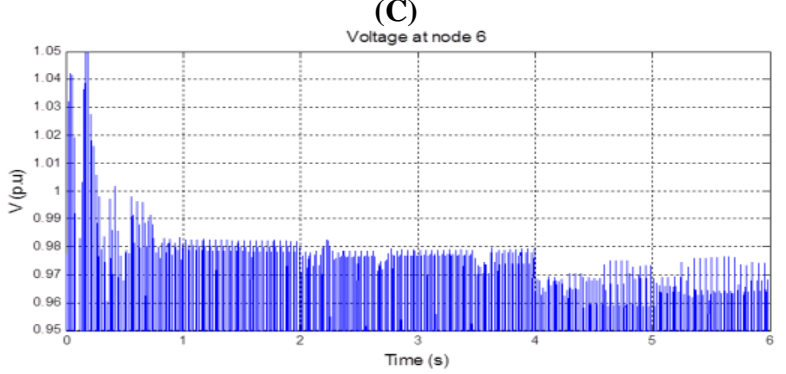

Fig. 24: Node 6 (A) FLC Decision (B) Load Power (C) Line Voltage.

The results show that the decision of each FLC will not be affected by the situation of the other one and there is no interaction between them. This means that there is no need for any type of coordination between the local controllers. This gives a very good advantage for this technique comparing with other solutions that depends on coordination and communication network.

\section{Conclusion}

This paper has presented a novel technique to regulate the voltage along a distribution feeder with PV integration. A local controller for each PV generator is designed using FL concepts to set the required consumption of reactive power for each inverter. The steadystate results have been presented which show the ability of the proposed control to handle the problem of voltage rise. Also, it offered a plug and play option for all customers on the feeder. Finally, the stability of the system has been assessed using MATLAB/Simulink with powersys tool box. The simulation results show that the system with FLC of the power factor responds in a stable manner to dynamic changed in load and PV power.

\section{References}

[1] R. A. Shalwala and J. A. M. Bleijs, "Voltage control scheme using Fuzzy Logic for residential area networks with PV generators in Saudi Arabia," in Innovative Smart Grid Technologies (ISGT), 2011 IEEE PES, 2011, pp. 1-6.

[2] J. D. Glover and M. S. Sarma, Power system analysis and design. Boston: PWS Pub., 1994.

[3] M. H. Nehrir and C. Wang. (2009). Modeling and control of fuel cells distributed generation applications.

Available:http://ieeexplore.ieee.org/xpl/bkabstractplus.jsp?bkn=544 3920 . 